\title{
Measuring secondary nucleation through single crystal seeding
}

\author{
Maria L. Briuglia ${ }^{1}$, Jan Sefcik ${ }^{1}$, Joop H. ter Horst ${ }^{2}$
}

${ }^{1}$ EPSRC Doctoral Training Centre in Continuous Manufacturing and Crystallisation, c/o Department of Chemical and Process Engineering, University of Strathclyde, James Weir Building, 75 Montrose Street, Glasgow, G1 1XJ, United Kingdom

${ }^{2}$ EPSRC Centre for Innovative Manufacturing in Continuous Manufacturing and Crystallisation, c/o Strathclyde Institute of Pharmacy and Biomedical Sciences, University of Strathclyde, Technology and Innovation Centre, 99 George Street, Glasgow, G1 1RD, United Kingdom.

\section{Abstract}

This article presents a novel assessment method for secondary nucleation rates using a wellcontrolled small scale seeding procedure. The procedure comprises of the seeding of a wellmonitored stirred supersaturated solution by a carefully selected single crystal under conditions at which spontaneous nucleation does not occur. The determined number of particles in time were translated to a suspension density using a calibration performed with monodisperse polymer spheres. The increasing crystal suspension density in time subsequently allowed the determination of the secondary nucleation rate under very specific conditions of supersaturation and temperature. The secondary nucleation rate was measured as a function of seed crystal size and supersaturation. It was observed that the time elapsed between the moment a single seed crystal is added and the moment the suspension density started to increase is larger when the seeded crystals are smaller and the supersaturation is lower. A systematic study of secondary nucleation at different supersaturations led to the determination of a supersaturation threshold for secondary nucleation, which could be used in industrial crystallization process design to identify process conditions with the right secondary nucleation rate behavior. 


\section{Introduction}

Crystallization is an intricate purification process that produces a final crystalline product through crystal nucleation, crystal growth and agglomeration ${ }^{1}$. Crystal nucleation is the formation of new small crystalline entities in a supersaturated liquid phase and, therefore, for a large part responsible for the final crystal size distribution ${ }^{2}$. Primary crystal nucleation occurs in a clear solution in the absence of crystalline material of its own kind ${ }^{3}$. Secondary nucleation, occurs as a result of the presence of crystals of the same compound in a supersaturated suspension $^{2,4}$. Thus, in case of secondary nucleation, parent crystals serve as catalyst for the formation of small new crystals ${ }^{5,6}$ The mechanism of this process of secondary nucleation is not well understood and is suggested to involve attrition ${ }^{7}$ or fluid $\operatorname{shear}^{8}$.Seeding is a well-known and often applied strategy to improve control over product size in batch-wise crystallization processes $^{9,10,11}$. Depending on the degree of supersaturation and on the position within the metastable zone, the seeding procedure results in crystal growth only while it avoids secondary and primary nucleation ${ }^{12}$. For continuous crystallization processes performed in cascades of continuous stirred tanks, which operate at high crystal slurry density and supersaturation, secondary nucleation represents the dominant mechanism for the generation of new crystals ${ }^{13}$. For continuous crystallization processes performed in plug flow type crystallizers such as the Continuous Oscillatory Baffled Crystallizer secondary nucleation has to be prevented in order to maintain control over the crystal size distribution ${ }^{14}$. With the adoption of continuous manufacturing in the pharmaceutical industry the ability to prevent, control and predict secondary nucleation is therefore increasingly important ${ }^{15}$.

Thus, in order to make knowledge based choices during the design of industrial crystallization processes, the secondary nucleation behavior has to be assessed under well controlled supersaturated conditions and seed crystal properties. The outcome of such an 
assessment would direct the design towards process conditions and crystal size distributions for which secondary nucleation can be exactly controlled. In addition, the assessment method would lead to valuable experimental data that can be used in the validation of secondary nucleation mechanisms.

Therefore, we aimed to develop a new approach to quantify secondary nucleation and to identify the secondary nucleation threshold within the metastable zone width. This approach uses a systematic single crystal seeding method in a small batch-wise crystallization process to follow the number density of crystals during the experiment, which determines the rate of secondary nucleation rate and provides the identification of supersaturation limits at which secondary nuclei can be formed and detected. We used Isonicotinamide (INA) in ethanol and Paracetamol (PAR) in 3-methyl-1-butanol (Isoamyl alcohol) as model compounds. For these compounds we studied the effect of seed crystal size and supersaturation ratio on the secondary nucleation rate.

\section{Experimental}

INA, PAR, isoamyl alcohol and ethanol, with a purity of $\geq 99 \%, 99 \%, 98 \%$ and $99.8 \%$ respectively, were obtained from Sigma-Aldrich.

\subsection{Metastable Zone Width determination}

Samples with various compositions were made by adding $1 \mathrm{~mL}$ of ethanol or isoamyl alcohol to a $1.5 \mathrm{~mL}$ vial containing a known amount of either INA or PAR. The $1.5 \mathrm{~mL}$ glass vials were tightly closed with a lid and sealed with para-film to avoid solvent evaporation and then placed in the Crystal16 (Technobis Crystallization Systems B.V.). The heating and cooling rates were set to $0.3^{\circ} \mathrm{C} / \mathrm{min}$ between -10.0 and $60.0^{\circ} \mathrm{C}$ for INA and 0.0 and $80.0^{\circ} \mathrm{C}$ for PAR in isoamyl alcohol. The samples were stirred at $700 \mathrm{rpm}$ using a PTFE coated magnetic stirring 
bar, which has a dimension of $1.3 \mathrm{~mm}$ and it was deposited at the bottom of the vial. The Metastable Zone Width (MSZW) is determined as the difference between clear point and cloud point temperature. The clear point temperature is the temperature at which the suspension upon heating turns into a clear solution, detected by the transmission of the light through the sample reaching a maximum. The temperature at which the clear solution, upon cooling, turns into a suspension, detected by a decrease in the transmission of light through the sample, is considered the cloud point temperature.

\subsection{Induction Time Distributions}

Stock solutions with different compositions of INA in ethanol and PAR in isoamyl alcohol were prepared and then samples from each stock solution were made by adding $3 \mathrm{~mL}$ of solvent to an $8 \mathrm{~mL}$ vial. The glass vials were tightly closed with a lid to prevent evaporation. A temperature recalibration of the Crystalline (Technobis Crystallization Systems B.V.) was performed to remove a small temperature difference between actual and set temperature. For each supersaturation value $S=C / C^{*}$ at $S=1.08$ and $1.12,40$ samples of INA in ethanol were tested; 12 samples were tested for the supersaturation values of $S=1.05$ and 1.19. Each sample was cooled to the temperature $T_{i}=18.0^{\circ} \mathrm{C}$ with a cooling rate $r=5^{\circ} \mathrm{C} / \mathrm{min}$. Furthermore, 12 samples were tested for 4 selected supersaturation values $(S=1.91,2.01,2.11$ and 2.20$)$ of PAR in isoamyl alcohol. Each sample was cooled to the temperature $T_{i}=20.0^{\circ} \mathrm{C}$ using a cooling rate $r=5^{\circ} \mathrm{C} / \mathrm{min}$. For both model systems, the moment the solution reached the temperature $T_{i}$, was taken as time zero for the induction time measurements. The sometimes observed fouling and crowning by others ${ }^{16}$ were not observed in this study.

\subsection{Single Crystal Seed Experiments}

\subsubsection{Preparation of supersaturated stock solutions}


All the stock solutions were prepared using the concentration reported in table 1 and ensuring the crystal dissolution by heating up to $20^{\circ} \mathrm{C}$ above the saturation temperatures $T_{s}$ for two hours on a hot stirrer plate at $500 \mathrm{rpm}$. Inside an incubator set at $T=60^{\circ} \mathrm{C}$ the stock solution was quickly filtered using a ceramic funnel and transferred to a pre-warmed bottle using a vacuum pump. The filtered hot stock solution was then divided over $248 \mathrm{ml}$ screw neck vials using a pre-warmed syringe. Each sample contained $3 \mathrm{~mL}$ of solution. A magnetic stirrer bar (VWR, micro $1 \times 0.3$ ) was added and the vials were tightly closed with a lid before they were located in the Crystalline at a stirrer speed of $700 \mathrm{rpm}$ for the bottom stirrer. From each stock solution 12 vials were prepared to perform seeded experiments and 12 for unseeded experiments.

Table 1: Stock solutions of differently concentrated solutions of INA in ethanol and PAR in isoamyl alcohol were prepared. The used concentrations $C$ having a saturation temperature $T_{s}$ and the solubility $C^{*}$ at the crystallization temperature $T_{x}$ determine the supersaturated ratio $S=C / C^{*}$ governing the single crystal seed experiments. The solubility $C^{*}$ of INA in ethanol at $T_{\mathrm{x}}=18^{\circ} \mathrm{C}$ and PAR in isoamyl alcohol at $T_{\mathrm{x}}=20^{\circ} \mathrm{C}$ is respectively $58.8 \mathrm{mg} / \mathrm{ml}$ and $27.5 \mathrm{mg} / \mathrm{ml}$.

\begin{tabular}{|c|c|c|c|c|}
\hline Sample & $\begin{array}{c}C \\
{[\mathrm{mg} / \mathrm{ml}]}\end{array}$ & $\begin{array}{c}T_{s} \\
{\left[{ }^{\circ} \mathrm{C}\right]}\end{array}$ & $\begin{array}{c}T_{x} \\
{\left[{ }^{\circ} \mathrm{C}\right]}\end{array}$ & $S$ \\
\hline \multirow{4}{*}{ INA in ethanol } & 62.0 & 18.6 & 18.0 & 1.05 \\
\hline & 63.6 & 19.2 & 18.0 & 1.08 \\
\hline & 66.0 & 20.3 & 18.0 & 1.12 \\
\hline & 70.0 & 21.9 & 18.0 & 1.19 \\
\hline \multirow{3}{*}{$\begin{array}{c}\text { PAR in } \\
\text { isoamyl alcohol }\end{array}$} & 52.7 & 30.0 & 20.0 & 1.91 \\
\hline & 55.5 & 32.5 & 20.0 & 2.02 \\
\hline & 58.2 & 35.0 & 20.0 & 2.12 \\
\hline
\end{tabular}




\begin{tabular}{|l|l|l|l|l|}
\hline & 60.4 & 37.5 & 20.0 & 2.20 \\
\hline
\end{tabular}

\subsubsection{Seeding experiments}

The Crystalline is a multiple reactor setup in which all 8 reactors can be independently controlled and monitored. The monitoring is done by the integrated cameras with a reported resolution of $2.8 \mu \mathrm{m} / \mathrm{pixel}$ and depth of field of $2.5 \mathrm{~mm}$. The recorded images are analyzed by internal software resulting in information on the crystal size distribution such as a particle count. For INA in ethanol a series of 8 vials were placed inside the reactors and heated to 40.0, 42.0, 44.0 and $45.0^{\circ} \mathrm{C}$ for each tested concentration reported in table 1 . The same procedure was applied to 4 vials of PAR in isoamyl alcohol. In this case the solutions were heated to $50.0,52.5,55.0$ and $57.5^{\circ} \mathrm{C}$ for the solution at $C=52.7,55.5,58.2$ and $60.4 \mathrm{mg} / \mathrm{ml}$ respectively. The vials were kept at these temperatures for 20 minutes in order to ensure a complete dissolution. Subsequently, a cooling rate of $r=1{ }^{\circ} \mathrm{C} / \mathrm{min}$ was applied to reach the desired temperature of $T=18.0^{\circ} \mathrm{C}$ for INA in ethanol and of $T=20.0^{\circ} \mathrm{C}$ for PAR in isoamyl alcohol. The time $t^{\prime}$ at which this temperature was reached is taken as the start of the experiment. A few minutes after, two crystalline vials were taken out from of the cooling coils and the lids were removed. One vial was seeded with a single crystal previously selected and characterized. The single crystal was added from a dry $1.5 \mathrm{ml}$ vial into the Crystalline vial containing the supersaturated solution. Then, quickly the vials were closed again with the lids and placed back in the cooling coils. Thus, both vials received the same treatment except for the addition of the seed crystal. The moment the vials are placed back inside the Crystalline represents time $t_{0}$. Different seed sizes were used for each supersaturation ratio. 
The particle count was monitored until it exceeded a value of about 200 particles at which the suspension density became too high to determine a reliable particles count.

\subsubsection{Seeds preparation and characterization}

A large amount of single crystals of INA in ethanol and PAR in isoamyl alcohol were formed using a combined cooling crystallization and solvent evaporation at room temperature. According to the determined MSZWs, the concentration of $C=90 \mathrm{mg} / \mathrm{ml}$ and $70 \mathrm{mg} / \mathrm{ml}$ were selected to crystallize INA in ethanol and PAR in isoamyl alcohol respectively. The samples of INA in ethanol were dissolved at $T=50^{\circ} \mathrm{C}$ and PAR in isoamyl alcohol at $T=70^{\circ} \mathrm{C}$. After a clear solution was obtained a cooling rate of $r=5^{\circ} \mathrm{C} / \mathrm{min}$ was applied until a temperature of $T=20^{\circ} \mathrm{C}$ was reached. Subsequently the temperature was kept constant. Well-defined single crystals with a plate-like shape were obtained for both APIs. Single crystals were isolated and shortly washed with a solution of pure cold ethanol for INA and pure cold isoamyl alcohol for PAR to remove any residues of materials and to avoid initial breading of attached small crystals during the seeding procedure. Microscope images were used for seed size characterization. All the crystals were sized circumscribing a circumference and calculating the projected area in $\mathrm{mm}^{2}$.

\subsubsection{Calibration of the view window volume in focus}

We want to determine the increase in the suspension number density $N_{\rho}$ due to secondary nucleation. The equipment we use can tell us a number $N$ of crystals in the suspension viewable by the camera. The relation between suspension number density $N_{\rho}$ and the number $N$ of crystals from the images was determined using different concentrations $C_{\mathrm{p}}$ of polystyrene microspheres (Sigma Aldrich) as is shown in figure 1. The product information provided by Sigma Aldrich reported that the used spheres have a monodisperse size of $d_{\mathrm{p}}=50$ 
nm and a density $\rho=1050 \mathrm{mg} / \mathrm{ml}$. The number $N$ of particles in focus were determined using the crystalline software from the average number of particles in 5 different pictures after 20 minutes of stirring. The deviation of $N$ between pictures at the same polystyrene microsphere concentration $C_{\mathrm{p}}$ is less than $10 \%$.

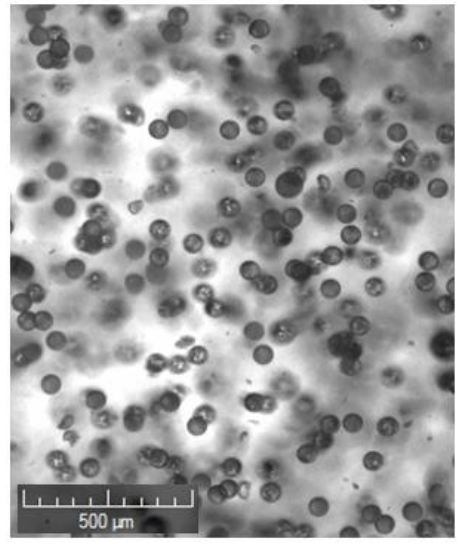

a)

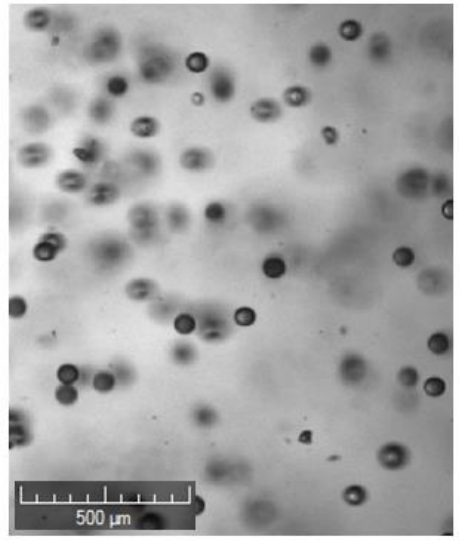

b)

Figure 1: Images from Crystalline software for different concentrations of polystyrene microspheres in water: a) A concentration $C_{\mathrm{p}}=3.37 \mathrm{mg} / \mathrm{ml}\left(N_{\rho}=25650 \# / \mathrm{ml}\right)$ led to a measurement of $N=96$ particles in focus, and b) A concentration of $C_{\mathrm{p}}=1.10 \mathrm{mg} / \mathrm{ml}\left(N_{\rho}=8400 \# / \mathrm{ml}\right)$ led to a measurement of $N=40$ particles in focus.

\section{Results}

First, secondary nucleation measurement conditions are established by measuring the metastable zone of the model compounds in the selected solvents and the induction time probability distribution for spontaneous nucleation. Then, the secondary nucleation induced by seeding a single crystal is studied for the two model compounds INA and PAR in supersaturated solutions in respectively ethanol and isoamyl alcohol using different single crystal seed sizes.

\subsection{Metastable Zone Width determination}

We want to measure secondary nucleation behavior under well-defined conditions at which primary nucleation is negligible and does not cloud the measurement. In order to establish 
these conditions, the metastable zone (MSZ) is measured in $1 \mathrm{ml}$ stirred solutions. The MSZ is defined as the zone between the solubility line and the MSZ limit. The MSZ usually is measured through obtaining clear and cloud point temperatures. Figure 2 shows the measured average clear and cloud points and the resulting MSZ for INA in ethanol and for PAR in isoamyl alcohol. The points are averages from three clear or cloud point temperature measurements, all showing a variation of less than $1.0^{\circ} \mathrm{C}$ per sample. Therefore, the error bars are smaller than the measurement symbols. The solubility line shows a good agreement with solubility data from literature ${ }^{15}$. 

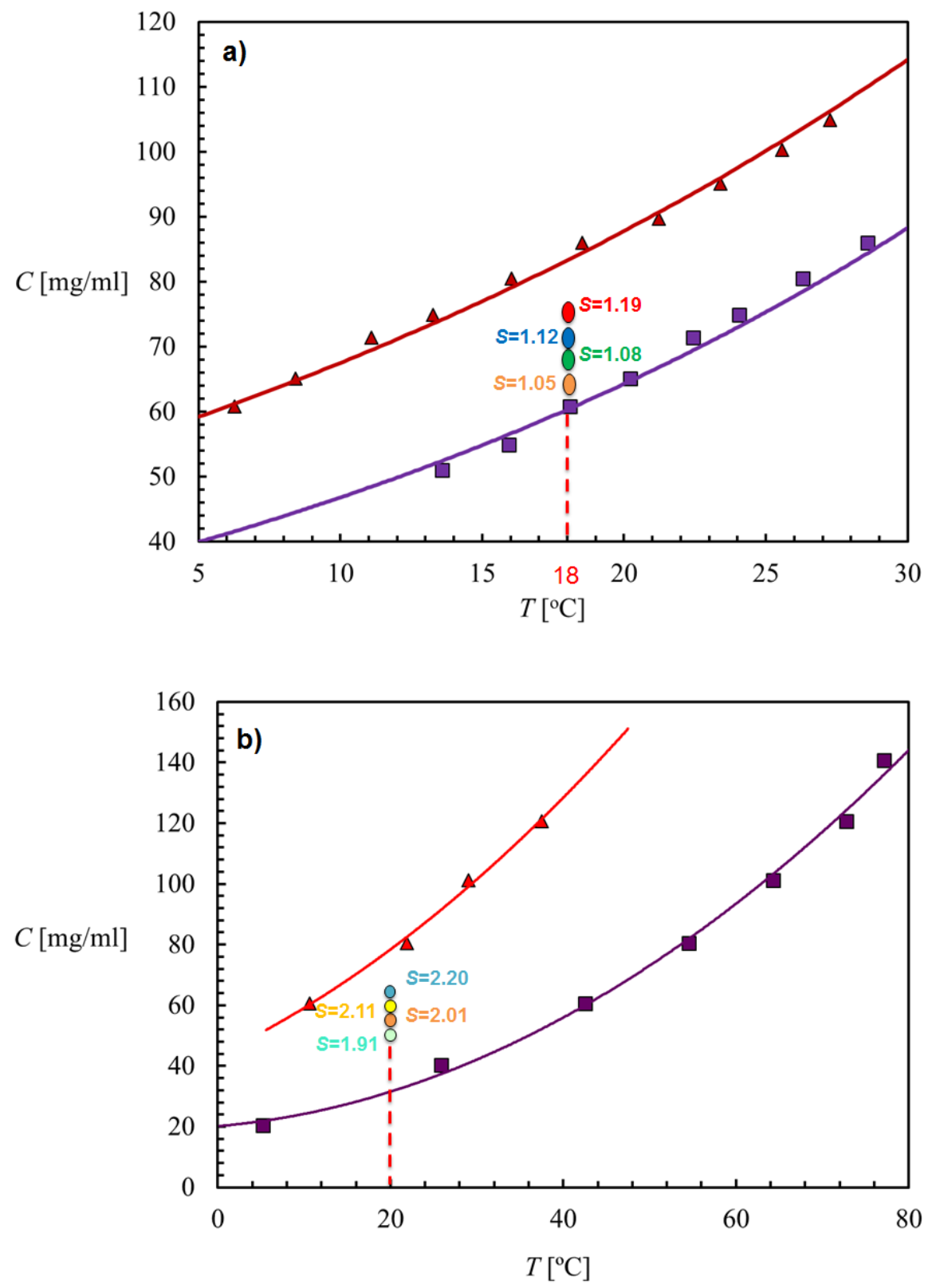

Figure 2: The Metastable Zone Width of INA in ethanol (a) and PAR in isoamyl alcohol (b) at different concentrations $C$. The purple curves in both figures indicate the solubility line constructed from the measured clear point temperatures (purple squares), while the red curve shows the metastable zone limit constructed from cloud point temperature averages at a specific concentration (red triangles). a) For INA in ethanol within the metastable zone four experimental concentrations at a temperature of $T=18.0^{\circ} \mathrm{C}$ were selected. The orange, green, blue and red dots indicate the single crystal seeding conditions at a supersaturation value of respectively $S=1.05,1.08,1.12$ and 1.19. b) For PAR in isoamyl alcohol four experimental concentrations at a temperature of $T=20.0^{\circ} \mathrm{C}$ were selected. The light green, orange, light yellow and light blue show the conditions for induction time measurements and single crystal seed experiments at supersaturation values of $S=1.91,2.01,2.11$ and 2.20 , respectively. 
The metastable zone width (MSZW) is the difference between clear $T_{s}$ and cloud $T_{c}$ point temperatures of the same sample. For instance, for a concentration of $60.8 \mathrm{mg} / \mathrm{ml}$ of INA in ethanol a clear point of $T_{s}=18.1^{\circ} \mathrm{C}$ and a cloud point of $T_{c}=6.3^{\circ} \mathrm{C}$ were measured, resulting in a MSZW of $11.8^{\circ} \mathrm{C}$ (figure $2 \mathrm{a}$ ). In the presented temperature range and with the used cooling rate of $0.3^{\circ} \mathrm{C} /$ minute, the average MSZW is around $10.0 \pm 1.1^{\circ} \mathrm{C}$.

The same procedure was followed to determine the MSZW for PAR in isoamyl alcohol (figure 2b). For instance, for a concentration of $61 \mathrm{mg} / \mathrm{ml}$ of PAR in isoamyl alcohol a clear point of $T_{s}=42.5^{\circ} \mathrm{C}$ and a cloud point of $T_{c}=10.6^{\circ} \mathrm{C}$ were detected resulting in a MSZW of $31.9^{\circ} \mathrm{C}$. This compound has a substantially larger MSZ in respect to that of INA in ethanol and it increases with temperature.

\subsection{Induction Time Measurements}

The solubility and metastable zone width allow the choice of conditions for the study of secondary nucleation by single crystal seeding of a supersaturated solution. First, a working temperature of $T=18.0$ and $20.0^{\circ} \mathrm{C}$ conveniently close to room temperature were chosen for INA in ethanol and for PAR in isoamyl alcohol, respectively. The solubility of INA in ethanol at $T=18.0^{\circ} \mathrm{C}$ is $60.5 \mathrm{mg} / \mathrm{mL}$, while that of PAR in isoamyl alcohol at $T=20.0^{\circ} \mathrm{C}$ is $27.5 \mathrm{mg} / \mathrm{ml}$. For INA in ethanol at $T=18.0^{\circ} \mathrm{C}$ the supersaturation ratios $S=1.05,1.08,1.12$ and 1.19 were chosen (figure 2a). For PAR in isoamyl alcohol at $T=20.0^{\circ} \mathrm{C}$ the supersaturation ratios $S=1.91,2.01,2.11$ and 2.20 were selected (figure 2b). At these temperatures the supersaturation conditions were expected to be sufficiently mild to minimize the possibility for spontaneous primary nucleation within the timeframe of the single crystal seeding experiment. 
Still, under these supersaturated conditions eventually primary nucleation will occur. In order to know the level of primary nucleation at these conditions the experimental induction time probability distribution in unseeded clear solutions were measured ${ }^{16}$. Figure 3 shows the obtained induction times of INA in ethanol at the supersaturations of $S=1.08$ and 1.12. In all 40 experiments crystals were detected at more than 59 and 37 minutes for the supersaturations of $S=1.08$ and $S=1.12$, respectively. After these times in increasingly more samples crystals appeared. The 12 induction times measured at $S=1.05$ and 1.19 show similar behavior.

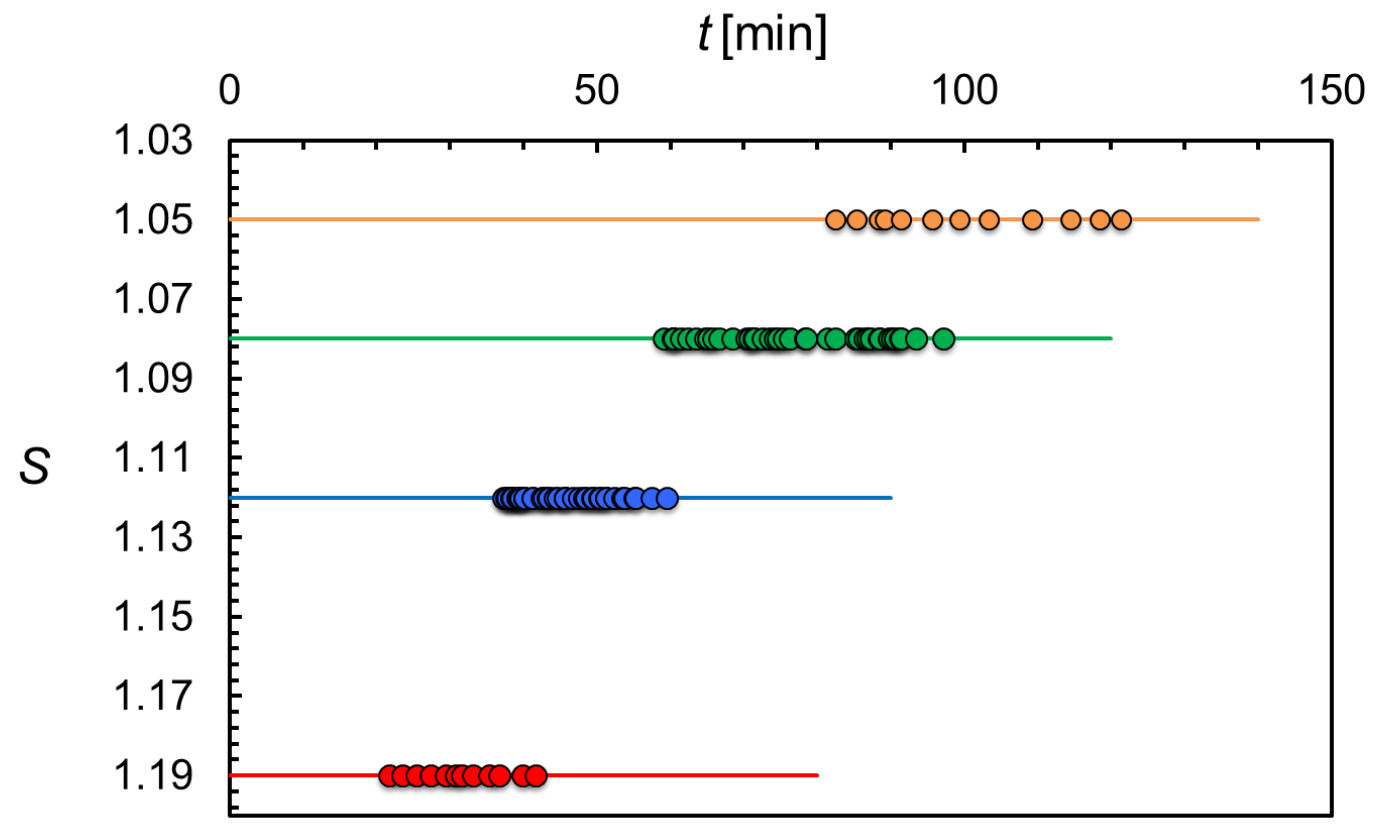

Figure 3: Experimental induction time measurements for 40 unseeded experiments of INA in ethanol at a supersaturation of $S=1.08$ (green dots) and 1.12 (blue dots) at a temperature of $T=18.0^{\circ} \mathrm{C}$. The lines represent the entire duration of the experiment. At the supersaturations $S=1.05$ and 1.1912 induction time measurements were done showing similar behavior.

The same analysis was conducted for PAR in isoamyl alcohol at the chosen supersaturation conditions. Figure 4 shows the obtained results of induction time $t$ as a function of supersaturation $S$. At the lowest investigated supersaturation $S=1.91$, the first sample 
nucleates 286 minutes after reaching the desired constant experimental conditions. At higher supersaturation the induction times reduce and at $S=2.20$ the shortest induction time is 170 minutes. These results for INA and PAR show that at the chosen conditions an extended interval of time exists during which primary nucleation is not observed. The chosen experimental conditions, reported in figure $2 \mathrm{a}$ and $2 \mathrm{~b}$ are therefore optimal conditions for studying the secondary nucleation rate of INA in ethanol and PAR in isoamyl alcohol.



Figure 4: Induction time measurements of PAR in isoamyl alcohol at four different supersaturations: $S=1.91$ (light green), 2.01 (orange), 2.11 (yellow) and 2.20 (light blue). At each supersaturation 12 experiments were conducted.

\subsection{Secondary Nucleation Rate Measurement Method}

\subsubsection{Suspension density calibration}

The used set-up allows the determination of the number of crystals $N$ in the viewing window of the camera representing an unknown part of the solution sample volume. Therefore, we performed a calibration to relate the suspension density $N_{\rho}$ to the measured number $N$ of 
particles using different concentrations of polystyrene microspheres. The used monodisperse polymer microspheres (figure 1) have a size of $d_{\mathrm{p}}=50 \mu \mathrm{m}$, giving them a particle volume of $V_{p}=\pi d_{p}{ }^{3} / 6=65.4 \times 10^{-9} \mathrm{ml}$. Therefore, a suspension of particles with a concentration $C_{p}$ in $\mathrm{mg} / \mathrm{ml}$ has a number density $N_{\rho}=C_{p} / V_{p} \rho=6 C_{p} / \pi \mathrm{d}_{\mathrm{p}}^{3} \rho$. For instance, for a concentration of $C_{p}=$ $1.10 \mathrm{mg} / \mathrm{ml}$, leading to a suspension density of $N_{\rho}=16 \times 10^{3} \# / \mathrm{ml}$, the number of particles in focus is measured to be $N=43$. Figure 5 shows the number density $N_{\rho}$ against the measured number $N$ of particles in focus. For an ideally mixed suspension the relation between $N$ and $N_{\rho}$ would be linear. The observed non-linearity therefore possibly indicates non-ideal mixing. Although we checked the particle count visually for many pictures, another source for the non-linearity is the software that determines the particle count from the pictures of the various suspension densities. We obtained our calibration through fitting of a $2^{\text {nd }}$ order polynomial through the data in figure 5 resulting in a relation between the measured number $N$ of crystals in focus and the suspension density $N_{\rho}$ :

$$
N_{\rho}=3.980 N^{2}+134.33 N+10.00
$$

This allows us to determine a suspension number density $N_{\rho}$ from the measured number $N$ of particles visible in the view window of the measurement device. We made sure not to extrapolate far outside the range of suspension densities $N_{\rho}$ measured. 


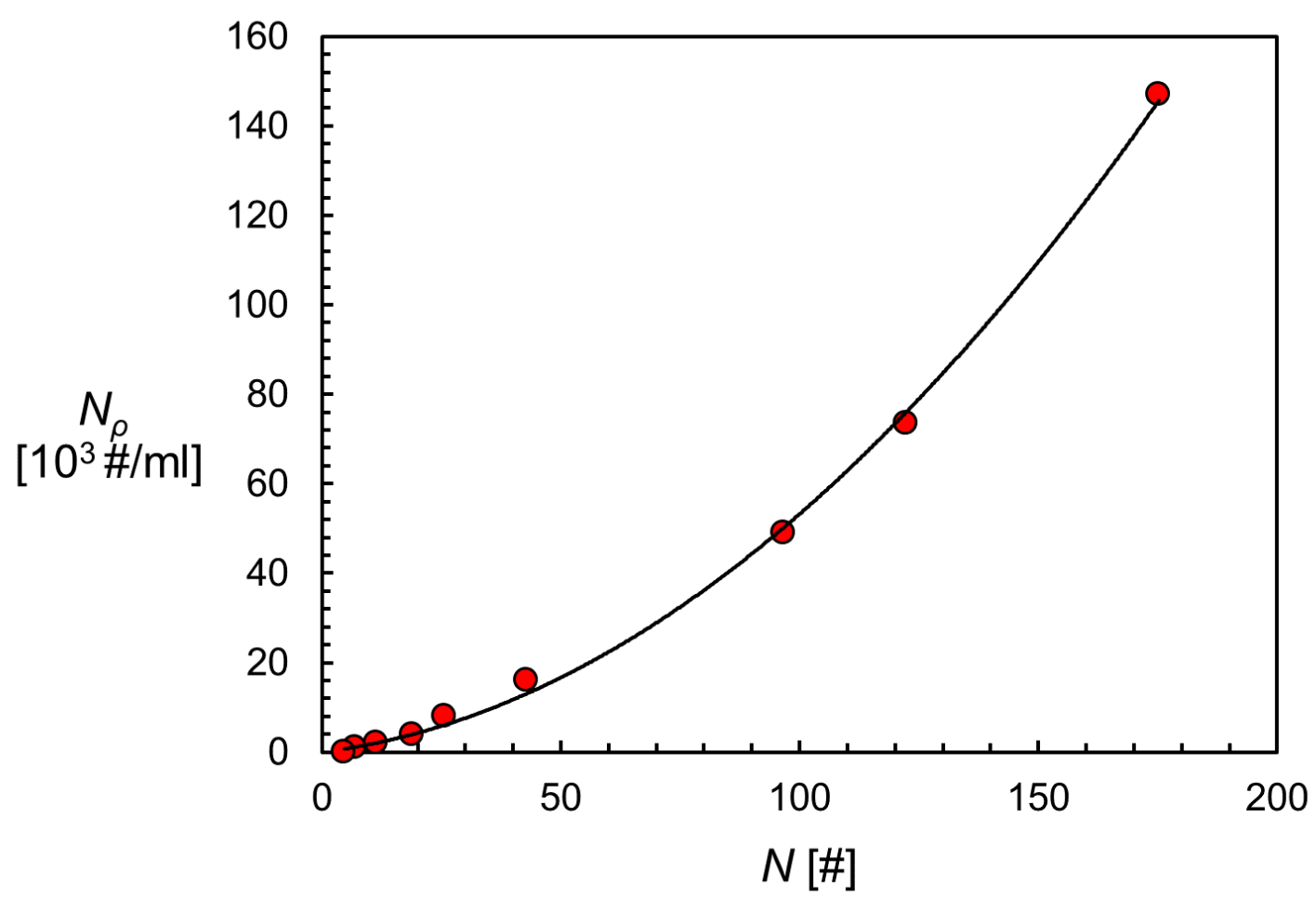

Figure 5: Calibration of suspension number density $N_{\rho}$ versus the number $N$ of particles in view of monodisperse polystyrene microspheres with diameter of $d=50 \mu \mathrm{m}$.

\subsubsection{Suspension Number Density}

Now that we established the process conditions under which primary nucleation is negligible (section 2.2) we can study secondary nucleation of INA in ethanol and PAR in isoamyl alcohol. We defined several times within the temperature profile used during a typical single seed crystal experiment for INA in ethanol (figure 6). Time $t^{\prime}$, represents the starting point of the experiment at which a constant temperature is reached. After time $t^{\prime}$ the solution is at a constant supersaturation and it is possible to seed the supersaturated solution with a single seed crystal. The moment the single crystal is added is defined as time $t_{0}$. The time difference $t_{0^{-}} t^{\prime}$ for the performed experiments in this study was never longer than 10 minutes. The time $t_{n}$ is the time from which primary nucleation can be expected to be detected. 


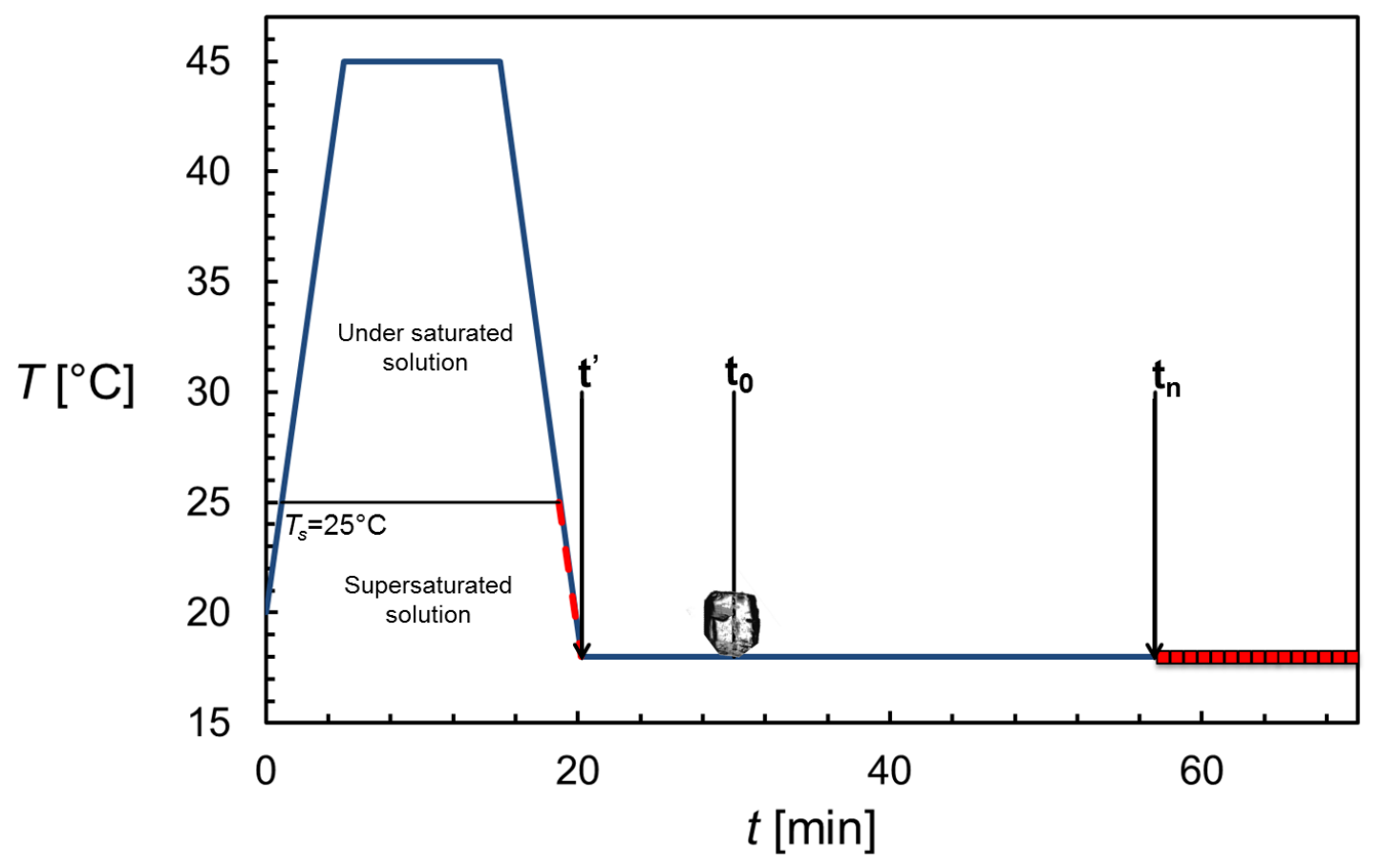

Figure 6: Definition of characteristic times during the single crystal seeding procedure for INA at $T=18.0^{\circ} \mathrm{C}$ in a supersaturated solution in ethanol at $S=1.08$. Time $t$ ' represents the time that isothermal conditions are reached; time $t_{0}$ is the moment that the single crystal is added; time $t_{n}$ is the smallest time measured under the same conditions for spontaneous primary nucleation in the absence of a seed crystal.

Figure 7 shows the suspension density $N_{\rho}$ during a single seed crystal experiment of INA in ethanol at $S=1.08$ and $T=18.0^{\circ} \mathrm{C}$ during two parallel experiments, a seeded (green symbols) and an unseeded one (red symbols), as a function of time $t$ - $t_{0}$ are presented in figure 7 . 


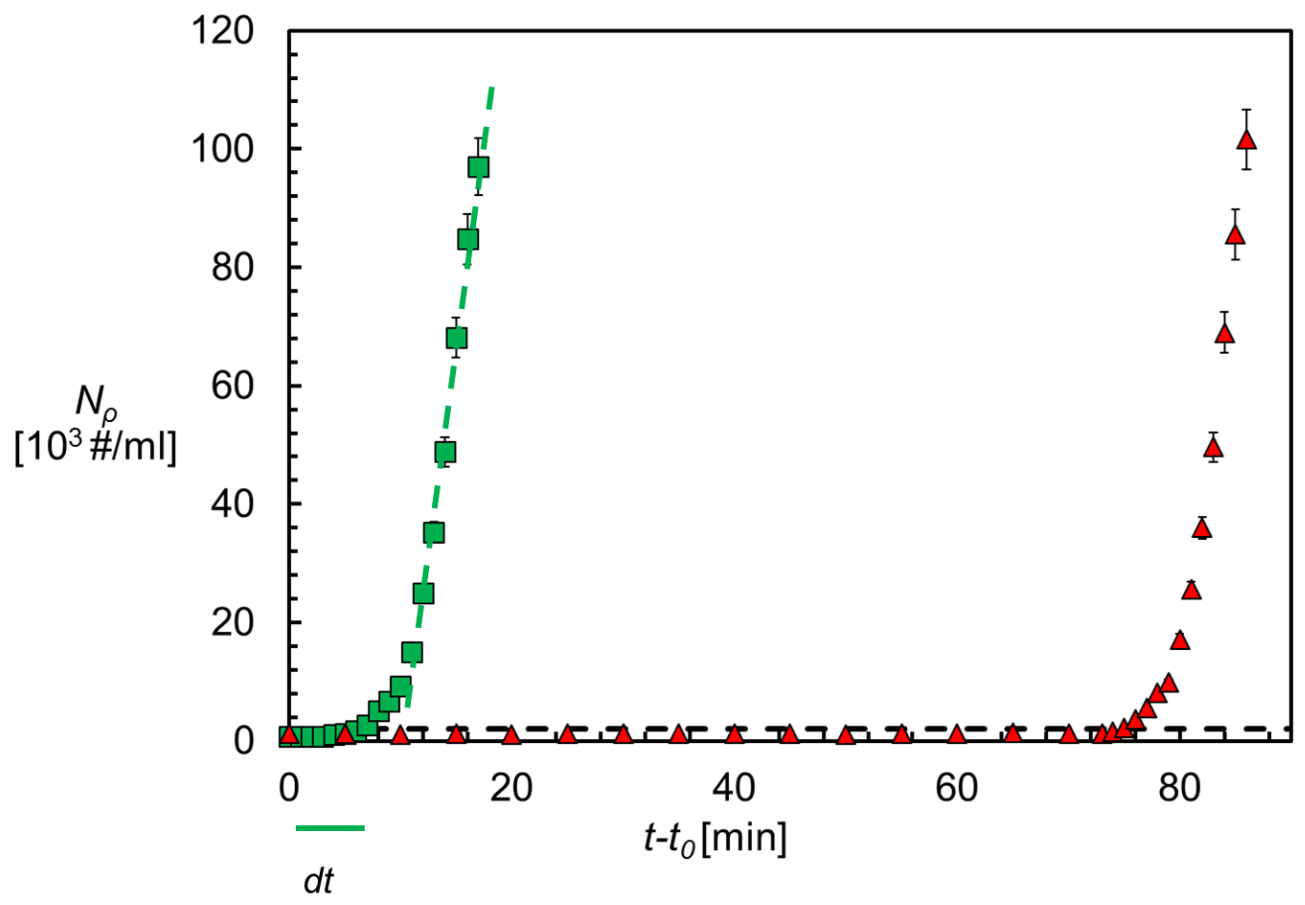

Figure 7: Suspension density $N_{\rho}(\# / \mathrm{ml})$ during a seeded experiment (green squares) and an unseeded experiment (red triangles) run in parallel for INA in ethanol at $S=1.08$ and $T=18.0^{\circ} \mathrm{C}$ as a function of $t-t_{0}$. For the seeded experiment, the solid line shows the delay time $t_{d}$, the green dashed line indicates the slope after secondary nucleation occurs and the dashed black line shows the cut-off value for the suspension density.

The seeded experiment shows a suspension density increase 6 minutes after the single seed crystal was added. In the unseeded experiment the suspension density raised only after 75 minutes, showing that spontaneous primary nucleation in the unseeded experiment is detected much later compared to secondary nucleation induced by the seed crystal in the seeded experiment. The suspension in the seeded experiment is therefore the result of secondary nucleation initiated by the seed crystal.

Before the rise, the measured suspension density fluctuates around a value of less than $2 \times 10^{3}$ \#/ml, which corresponds to a fluctuation of 5-9 particles in focus. This is due to impurity particles such as dust particles present in the samples. In order to properly and consistently identify a suspension density rise, it was assumed that a suspension density larger than $2 \times 10^{3}$ $\# / \mathrm{ml}$ indicated the presence of crystals. The first count after this value of $2 \times 10^{3} \# / \mathrm{ml}$ represents the delay time in the experiment. The delay time $t_{d}$ is interpreted as the time 
needed for the parent crystal to undergo secondary nucleation. The delay time in the single crystal seed experiment in figure 7 was determined to be $t_{d}=6 \mathrm{~min}$.

The upper limit for this method is set at a suspension density of $N_{\rho}=100 \times 10^{3} \# / \mathrm{ml}$; above this value, the suspension density becomes too large to discriminate between the single particles in the determination of number $N$ of particles in focus. Therefore, the seeded experiment presented in figure 7 shows a suspension density rise up to around $N_{\rho}=97 \times 10^{3} \# / \mathrm{ml}$ volume after 18 minutes of experiment.

\subsection{Secondary Nucleation Rate}

Between the established upper and lower limits of the suspension number density $N_{\rho}$ it was observed that the rise was roughly linear in time, see for instance figure 7 . The secondary nucleation rate $B$ was therefore determined from the slope of a fitted straight line between the upper and lower limits of the suspension density. For the seeded experiment in figure 7 the secondary nucleation rate was determined to be $B=9.4 \times 10^{3}$ particles $/ \mathrm{ml} / \mathrm{min}$.

This single crystal seeding method now allows us to measure secondary nucleation rates under specific conditions of temperature and supersaturation and as a function of seed crystal properties such as single crystal seed size. Different seed sizes with a square root of the projected area between 0.45 and $3 \mathrm{~mm}$ were used to determine secondary nucleation rates in single crystal seed experiments. Figure 8 shows how the suspension density develops for single crystal seed experiments at a supersaturation $S=1.12$ using these differently sized seed crystals. Clearly, the seed crystal size influences the secondary nucleation rate. A larger single crystal seed (e.g., blue squares in figure 8) produces a faster rise in the suspension density leading to a higher secondary nucleation rate. 


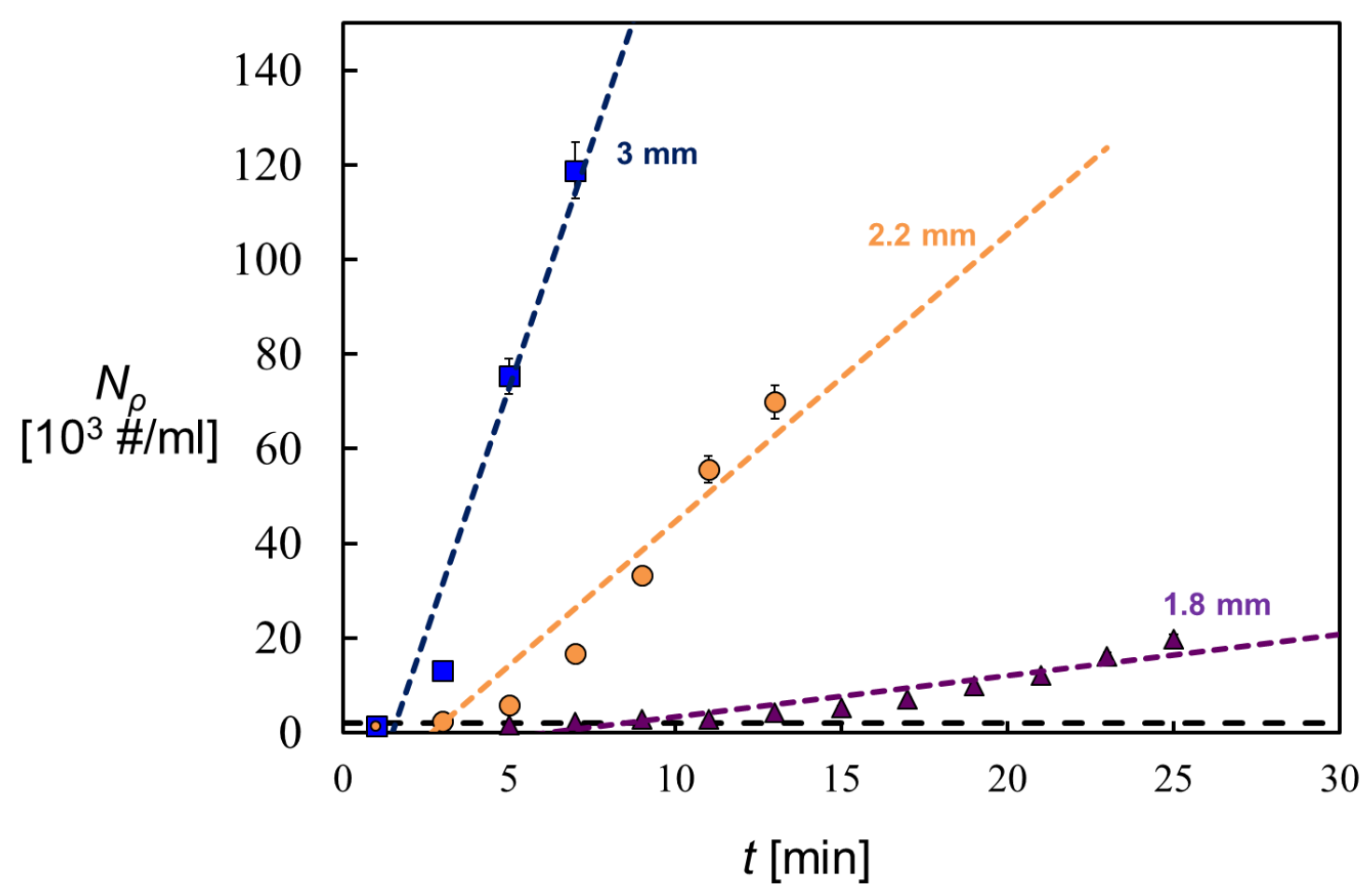

Figure 8: Suspension Number density $N_{\rho}$ against time $t$ of single crystal seed experiments for INA in ethanol at a supersaturation $S=1.12$. Different colors of the symbols represent different single crystal seed size: purple triangles $(1.3 \mathrm{~mm})$, orange circles $(2.2 \mathrm{~mm})$, and blue squares $(3 \mathrm{~mm})$. The horizontal black dashed line represents the cut-off value for the suspension density count for secondary nucleation determination.

The measured delay time $t_{d}$ also depends on the parent crystal size. This delay time decreases if the seeded crystal is larger, as shown in figure 8 . The detected delay time, for a seed crystal size of $1.8 \mathrm{~mm}$, results in $t_{d}=9$ minutes; while when the seeded crystal is $3 \mathrm{~mm}$, the delay time is reduced to $t_{d}=3$ minutes.

The same procedure was conducted for single crystal seeding of PAR in isoamyl alcohol. Figure 9 shows the results for the suspension density $N_{\rho}$ during the run of the experiments for four different investigated supersaturations. The lower supersaturation $S=1.91$ indicated by the orange symbols presents a slower increasing in suspension density $N_{\rho}$ resulting in a lower secondary nucleation rate. At lower supersaturation $S=1.91$ the delay time $t_{\mathrm{d}}$ increases. When 
supersaturation increases, the number of particles increases faster and secondary nucleation rate is higher.

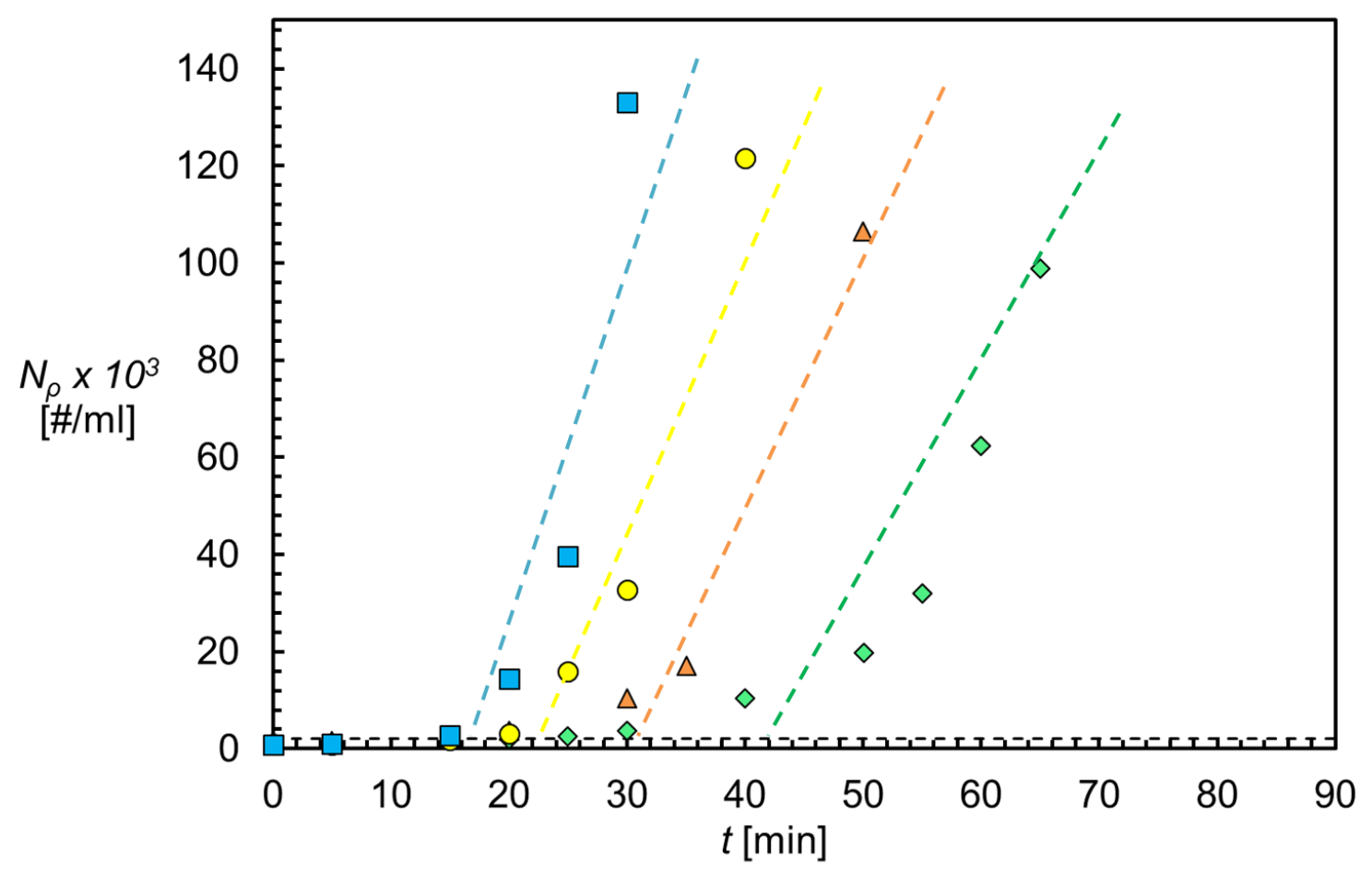

Figure 9: Single Crystal Seed Experiments of PAR in isoamyl alcohol. Suspension density $N_{\rho}$ is plotted against the time $t$ for secondary nucleation determination. Four supersaturation ratios were investigated: $S=1.91$ (light green with rhombic symbols), 2.01 (orange with triangles symbols), 2.11 (yellow with circles symbols) and 2.20 (light blue with squares symbols). The used seeded crystals had a range size between 1.5-2.8 mm.

Secondary nucleation rates for INA in ethanol using different seed sizes and at $S=1.05,1.08$, 1.12 and 1.19 are presented in figure $10 \mathrm{a}$, while the secondary nucleation rates $B[\# / \mathrm{ml} / \mathrm{min}]$ for PAR in isoamyl alcohol are reported for different seed sizes at $S=1.91,2.01,2.11,2.20$ in figure 10b. Although the rise in suspension density for PAR in isoamyl alcohol deviates from linear behavior with time the slope was continued to be used as estimate for the secondary nucleation rate. The results for both compounds show that the conducted experiments using a larger seed size on average results in a larger secondary nucleation rate at the same 
supersaturation. Although in figure 10a for INA in ethanol the obtained secondary nucleation rates for $S=1.05$ and 1.08 are quite similar, on average an increase in supersaturation leads to an increase in the secondary nucleation rate.


Figure 10: a) Secondary nucleation rate $B$ of INA in ethanol [\#x $10^{3} / \mathrm{ml} / \mathrm{min}$ ] as a function of seed size $L$ for supersaturations $S=1.05$ (orange rhombs), 1.08 (green circles), 1.12 (blue triangles) and 1.19 (red squares) at temperature $T=18^{\circ} \mathrm{C}$. For each supersaturation 12 different seeds sizes between 0.4 and $3 \mathrm{~mm}$ were used. The blue window indicates a range of seed sizes between 1.7 and $2.4 \mathrm{~mm}$ that were used to compare more in details the obtained nucleation rates. b) Secondary nucleation rate $B$ of PAR in isoamyl alcohol $\left[\times 10^{3} / \mathrm{ml} / \mathrm{min}\right]$ as a function of seed size $L$ for supersaturations $S=1.91$ (light green rhombs), 2.01 (orange triangles), 2.11 (yellow circles) and 2.20 (light blue squares) at temperature $T=18^{\circ} \mathrm{C}$. For each supersaturation 4 different seeds sizes between 0.3 and $4.2 \mathrm{~mm}$ were used. The blue window indicates a range of seed sizes between 2.0 and $2.6 \mathrm{~mm}$ that were used to compare more in details the obtained nucleation rates.

\section{Discussion}

The single crystal seed experiments allowed measurements of secondary nucleation rates. For INA in ethanol (figure 10a), using the same single crystal seed size, the secondary nucleation rate increases with increasing supersaturation. Considering experiments using a single crystal seed size of 1.7-2.4 mm, the determined secondary nucleation rates $B$ are compared at different supersaturations in figure 11 in which for each supersaturation 3 measurements are performed for INA in ethanol (figure 11a) and one for PAR in isoamyl alcohol (figure 11b).

In figure 11a, at $S=1.05$ (orange points), the number at the end of the experiment raises to only 30 particles resulting in an average secondary nucleation rate $B=2.0 \times 10^{3} \# / \mathrm{ml} / \mathrm{min}$ over 
three experiments. The same analysis is conducted for secondary nucleation rates at $S=1.08$, 1.12 and 1.19. The graph shows that the secondary nucleation rate $B$ increases with supersaturation. Furthermore, by extrapolating the line through the secondary nucleation rate data points, a secondary nucleation threshold $\mathrm{S}_{\Delta}$ at a supersaturation value of around $S=1.033$ is detected. Below this supersaturation value, the seeded crystal will just grow without inducing secondary nucleation.
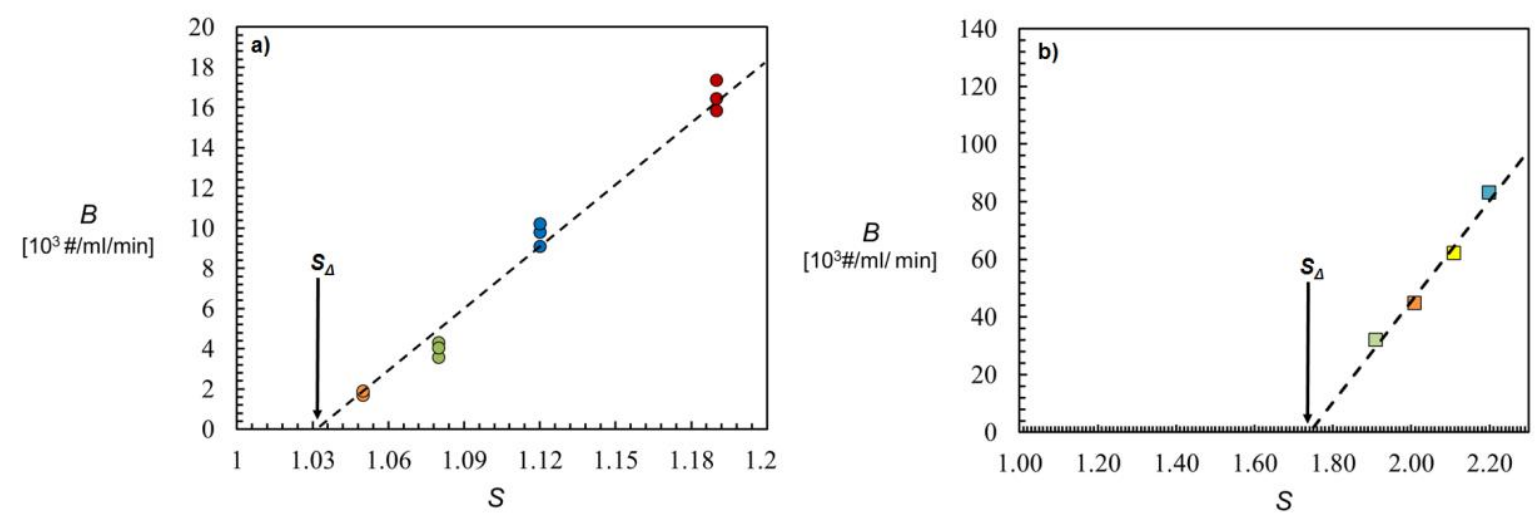

Figure 11: The secondary Nucleation Threshold $\mathrm{S}_{\Delta}$ determined from the single crystal seed experiments of INA in ethanol (a) and PAR in isoamyl alcohol (b). a) Only the secondary nucleation rate data generated with seed sizes of 1.7-2.4 mm is used. The secondary nucleation rate $B$ was measured at four supersaturated conditions: $S=1.05$ (orange dots), 1.08 (green dots), 1.12 (blue dots) and 1.19 (red dots). The arrows indicate the obtained value of the secondary nucleation threshold $\mathrm{S}_{\Delta}=1.033$. b) The single seeded crystals for PAR in isoamyl alcohol had a size range of 2-2.6 $\mathrm{mm}$. The secondary nucleation rate $B$ was measured at four supersaturated conditions: $S=1.91$ (light green dot), 2.01 (orange square), 2.11 (yellow square) and 2.20 (light blue square). The arrow indicates the obtained value of the secondary nucleation threshold $\mathrm{S}_{\Delta}=1.72$.

The same procedure can be applied to determine the secondary nucleation threshold $\mathrm{S}_{\Delta}$ for PAR in isoamyl alcohol (figure 11b). In this case the size of the used seed crystals was between 2-2.6 mm for each supersaturation was conducted. Each colored square indicates the obtained nucleation rate $B$ at a specific value of supersaturation: $S=1.9$ (light green), 2.0 (orange), 2.1 (yellow) and 2.2 (light blue). The Secondary Nucleation Threshold was determined to be $S_{\Delta}=1.73$. The SNT for PAR in isoamyl alcohol is substantially higher than 
that for INA in ethanol, showing that INA in ethanol is substantially more susceptible to secondary nucleation than PAR in isoamyl alcohol.

As for all nucleation detection techniques ${ }^{17,18}$, also in this methodology there are limitations for detection of newly formed crystals. In our set up, the early born crystals are easily recognized since the lower detection limit of particles is around $3 \mu \mathrm{m}$. The occurrence of agglomeration, which was not observed in our experiments, would cause problems for the counting procedure therefore affecting the secondary nucleation rate determination. Additionally, if the suspension density becomes too large it is not possible to recognize the individual crystals and further secondary nucleation rate measurements cannot be done. In our case the measurement had to be stopped when the suspension density $N_{\rho}>140 \times 10^{3} \mathrm{\#} / \mathrm{ml}$. It is interesting to note the similarity in the particle count behavior in the seeded and unseeded experiments in figure 7. The similarity of seeded and unseeded experiments might be an indication that the underlying mechanisms in these crystallizing solutions are the same. This seems to show then that also in the unseeded experiment a single crystal is responsible for the formation of the entire suspension and that the unseeded experiment follows the single nucleus mechanism ${ }^{17}$, possibly involving secondary nucleation through attrition rather than shear. Once the primary nucleated single crystal is sufficiently large, it induces secondary nucleation by attrition of the single crystal, generating a similar particle count behavior as in the case of the single crystal seeded experiment.

Currently, secondary nucleation has mainly been described through empirical power law models, relating observed nucleation rates to operational parameters. ${ }^{19}$ Although elaborate mechanistic models are available for attrition-induced secondary nucleation ${ }^{20,21}$ these are not validated through experiments. For contact secondary nucleation a semi-empirical model has been suggested ${ }^{22,23}$. The developed secondary nucleation rate measurement method enables a 
systematic study of secondary nucleation kinetics and the validation of new secondary nucleation theories.

Our novel seeding procedure can be incorporated in crystallization workflow procedures ${ }^{24}$ allowing rapid development of industrial crystallization processes. The series of steps for a systematic procedure for secondary nucleation determination are presented in figure 12 . The described workflow allows for a rational discrimination between primary and secondary nucleation events. It consists of 6 stages and starts from the basic knowledge of solubility and MSZW (stage 1) in order to define the crystallization window for the compound under investigation. Once the MSZW is determined, the first decision needs to be made: the desired supersaturation ratios within the MSZW for the induction time measurements will have to be chosen (stage 2). The levels of supersaturation need to be sufficiently close to the solubility curve in order to facilitate the procedure for seeding and avoid unwanted primary nucleation. Spontaneous nucleation can be monitored through induction time measurements (stage 3). After characterization of the seeds (stage 4) they can be used in the single crystal seed experiments for secondary nucleation rate determination (stage 5). The procedure is repeatable at different supersaturation ratios, which allows the secondary nucleation threshold $\Delta$ determination (stage 6). This threshold can be used in the industrial crystallization design process to either avoid or enhance secondary nucleation, taking into account that fluid dynamics in the experiments and under industrial conditions might be substantially different, possibly influencing this secondary nucleation threshold. 


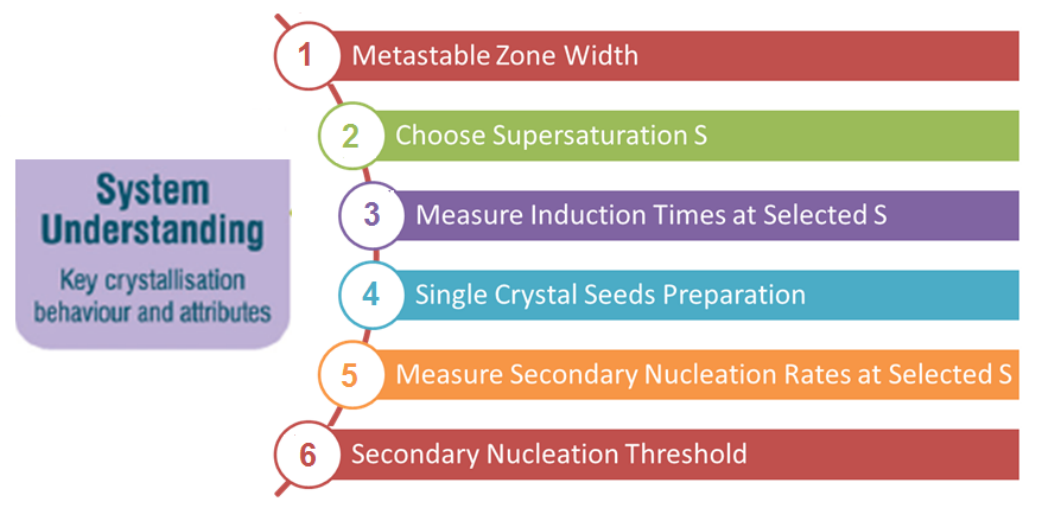

Figure 12: Workflow for secondary nucleation, which allows system understanding. Stage 1: The solubility and the MSZW are the starting points to identify the conditions to crystallize the material at the selected concentration and temperature. Stage 2: The supersaturation ratios for secondary nucleation studies are selected within the MSZW. Stage 3: Induction time experiments at the selected supersaturation ratios are required in order to determine the time frame for seeding experiments avoiding unwanted primary nucleation. Stage 4: The crystals that will be used as seeds need to be well-characterized. Stage 5: The conditions for the secondary nucleation experiments are established and now the seeded crystals can be added and secondary nucleation can be monitored at the selected conditions of supersaturation. Stage 6: Analyzing the secondary nucleation rates at different supersaturated conditions in order to determine the secondary nucleation threshold $S_{\Delta}$ understanding at which supersaturation ratio secondary nucleation is negligible. 


\section{Conclusions}

A meticulous and reproducible single crystal seeding approach in batch-wise cooling crystallisation has been developed allowing the study of secondary nucleation. Upon seeding a well characterized single parent crystal in an accurately controlled supersaturated solution, the suspension density starts increasing after a delay time allowing the determination of the secondary nucleation rate. The secondary nucleation rate measurements are affected by the seed crystal size: a larger parent crystal produces more secondary nuclei per unit of time and triggers the secondary nucleation process more quickly. The determination of secondary nucleation rates as a function of supersaturation ratio shows a supersaturation threshold for secondary nucleation below which secondary nucleation is negligible. This threshold, which is also a function of seed size, is a valuable characteristic that can be used in crystallization process design. The developed approach will further be valuable in elucidating secondary nucleation rate mechanisms.

\section{Acknowledgements}

The authors would like to thank EPSRC, Centre for Innovative Manufacturing in Continuous Manufacturing and Crystallisation (Grant Ref EP/K503289/1) for funding this work. The authors would like to acknowledge that this work was carried out in the CMAC National Facility supported by UKRPIF (UK Research Partnership Fund) award from the Higher Education Funding Council for England (HEFCE) (Grant Ref HH13054).

\section{References}

1) Nyvlt, J. Nucleation and Growth Rate in Mass Crystallization. Prog. Crystal Growth 
and Charact. 1984, 9, 335-370.

2) Mullin, J. W. Crystallization, $4^{\text {th }}$ ed.; Butterworth-Heinemann: London, 2001.

3) Davey, R., Schroeder, S.L.M., ter Horst, J.H.. Nucleation of Organic Crystals-A molecular prospective. Angewandte Chemie. 2013, 52, 2166-2179.

4) Meadhra, R.O, Kramer, H.J.M, Van Rosmalen, G.M., 1996. Model for Secondary nucleation in a suspension crystalliser, AIChE, 42, 972-982.

5) Myerson, A. S. Handbook of Industrial Crystallization, 2nd ed.; ButterworthHeinemann: Woburn, MA, 2002.

6) Frawley, P.J., Mitchell, N.A., O'Ciardha, C.T., Hutton, K.W. The effects of supersaturation, temperature, agitation and seed surface area on the secondary nucleation of paracetamol in ethanol solutions. Chemical Engineering Science. 2012, $75,183-197$.

7) Gahn, C., Mersmann, A. Brittle fracture in crystallization processes Part A. Attrition and abrasion of brittle solids. Chemical Engineering Science. 1999, 54, 1273-1282.

8) Callahan, J. C., Ni, X.W. An investigation into the effect of mixing on the secondary nucleation of sodium chlorate in a stirred tank and an oscillatory baffled crystallizer. CrystEngComm. 2015, 16, 690-697.

9) Adi, H., Larson, I., Stewart, P., 2007. Use of milling and wet sieving to produce narrow particle size distributions of lactose monohyrate in the sub-sieve range. Powder Technol. 179, 95-99.

10) Jagadesh, D., Kubota, N., Yokota, M., Doki, N. Seeded effect on Batch Crystallisation of Potassium Sulfate under Natural Cooling mode and a simple Design Method of Crystallizer. J. Chem. Eng. Jpn. 1999, 32, 514-520.

11) Kalbasenka, A.N., Spierings, L.C.P., Huesman, A.E.M., Kramer, H.J.M. Application 
of seeding as a process actuator in a model predictive control framework for fed-batch crystallisation of afmmonium sulphate. Part. Part. Syst. Charact. 2007, 24, 40-48.

12) Kubota, N., Doki, N., Yokota, M., Sato, A. Seeding policy in batch cooling crystallisation. Powder Technol. 2001, 121, 31-38.

13) Ludwick, J.C., Henderso, P.L.. Particle shape and inference of size from sieving. Sedimentology. 1968, 11, 197-235.

14) Lung-Somarriba, B.L.M., Moscosa-Santillan, M., Porte, C., Delacroix, A. Effect of seeded surface area on crystal size distribution in glycine batch cooling crystallisation: a seeding methodology. J Cryst. Growth. 2004, 270, 624-632.

15) Kobari, M., Kubota, N., Hirasawa, I. Secondary nucleation mediated effects of stirrer speed and growth rate on induction time for unseeded solution. Cryst.Eng.Comm. 2012, 14, 5255- 5261

16) Jiang, S., ter Horst, J.H. Crystal nucleation rates from probability distributions of induction times. Cryst. Growth Des. 2011, 11 (1), 256-261.

17) Kadam, S.S., Kramer, H.J.M., ter Horst, J.H. Combination of a Single Primary Nucleation Event and Secondary Nucleation in Crystallization Processes. Cryst. Growth Des. 2011, 11, 1271-1277.

18) Kulkarni, S.A; Kadam, S.S; Meekes, H.; Stankiewicz, A.I.; ter Horst, J.H. Crystal Nucleation Kinetics from Induction Times and Metastable Zone Widths. Cryst. Growth Des. 2013, 13, 2435-244.

19) Ulrich, J., and Strege, C. Some aspects of the importance of metastable zone width and nucleation in industrial crystallizers. J. Cryst. Growth 2002, 237-239, 21302135.

20) Agrawal, S.G., Paterson, A.H.J. Secondary Nucleation: Mechanisms and Models, 
Chemical Engineering Communications. 2015, 202, 698-706.

21) Beckmann, W., Nickisch, K., Budde U. Development of a Seeding Technique for the Crystallization of the Metastable A Modification of Abecarnil. Organic Process Research \& Development. 1988, 2, 298-304.

22) Chianese, A., Sangl, R.G., Mersmann, A.B. On the size distribution of fragments generated by crystal collisions. Chemical Engineering Communication. 1996, 1, 146.

23) Garside, J., Rusli, I.T., Larson, M.A. Origin and size distribution of secondary nuclei. AIChE. 1979, 25, 57.

24) Brown, C., McGlone, T., Yerdelen, S., Srirambhatla, V., Mabbott, F., Gurung, R., Briuglia, M.L., Ahmed, B., Polyzois, H., McGinty, J., Perciballi, F., Frysikopolous, D., MacFhionnghaile, P., Siddique, H., Raval, V., Harrington, T.S., Vassileiou, A.D., Robertson, M., Prasad, E., Johnston, A., Johnston, B., Nordon, A., Srai, J.S., Halbert, G. , ter Horst, J.H., Price, C.J., Rielly, C.D. , Sefcik, J. , Florence, A. Enabling Precision Manufacturing of Active Pharmaceutical Ingredients: Workflow for Seeded Cooling Continuous Crystallisations. Molecular Systems Design Engineering. 2018, $3,518-549$. 


\section{For Table of Contents Use Only}

Title: Measuring secondary nucleation through single crystal seeding

List of authors:

Maria L. Briuglia,

Jan Sefcik,

Joop H. ter Horst

\section{Toc graphic:}



Suspension density $N_{\rho}(\# / \mathrm{ml})$ during a single crystal seed experiment (green squares) and an unseeded experiment (red triangles) run in parallel for INA in ethanol at $S=1.08$ and $T=18.0^{\circ} \mathrm{C}$ as a function of time $t-t_{0}$ proceeded sincethe seed crystal is added in the single crystal seed experiment.

\section{Toc synopsis:}

This paper offers a new approach to systematically study secondary crystal nucleation. The procedure includes a controlled seeding procedure with a well-characterized single crystal. 
By measuring the number of secondary nuclei formed, the secondary nucleation rate at different supersaturation ratios and different seeds size can be monitored determining the secondary nucleation threshold for the exanimated system. 